\title{
Lessons from implementation science: Key to curbing the global HIV epidemic
}

Julie Pulerwitz

Population Council

\section{Recommended Citation}

Pulerwitz, Julie. 2021. "Lessons from implementation science: Key to curbing the global HIV epidemic," presentation at DC Center for AIDS Research (DC CFAR) Citywide Seminar, 22 April. 


\section{POPULATION COUNCIL}

Ideas. Evidence. Impact.

\section{LESSONS FROM}

IMPLEMENTATION

SCIENCE:

KEY TO CURBING THE GLOBAL HIV EPIDEMIC

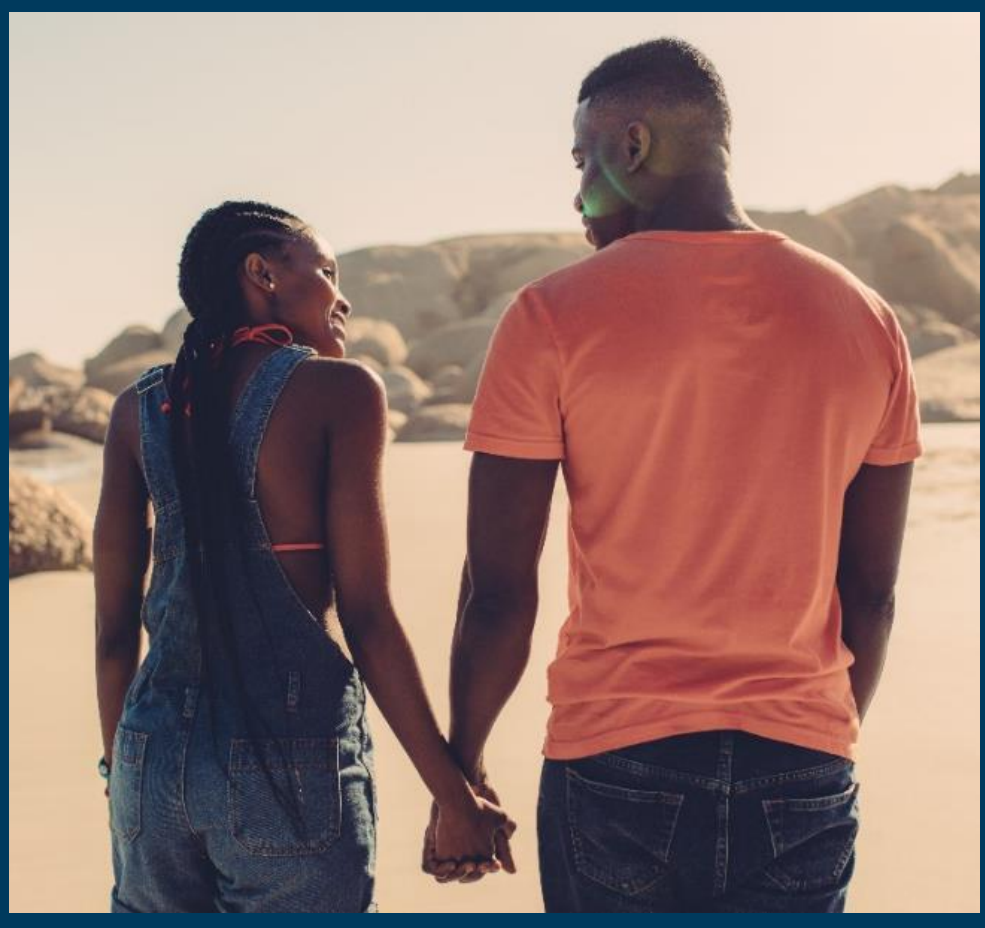

Julie Pulerwitz, ScD

HIV Program Director

DC Center for AIDS Research (DC CFAR) Citywide Seminar 


\section{Global HIV epidemic}

- About 38 million people living with HIV (PLHIV) worldwide

- Out of UNAIDS treatment 90-90-90 goals for 2020:

- 79\% knew status

- 62\% access antiretroviral therapy (ART)

- 53\% virally suppressed

- Prevention? 1.7 million people acquired HIV in the past year

- 940,000 people died from HIV-related illness in last year

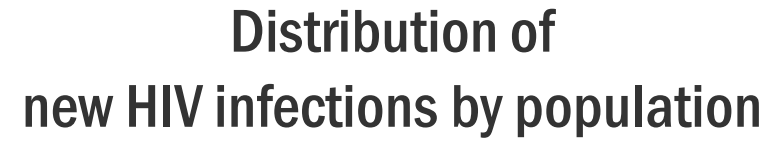

Sex workers

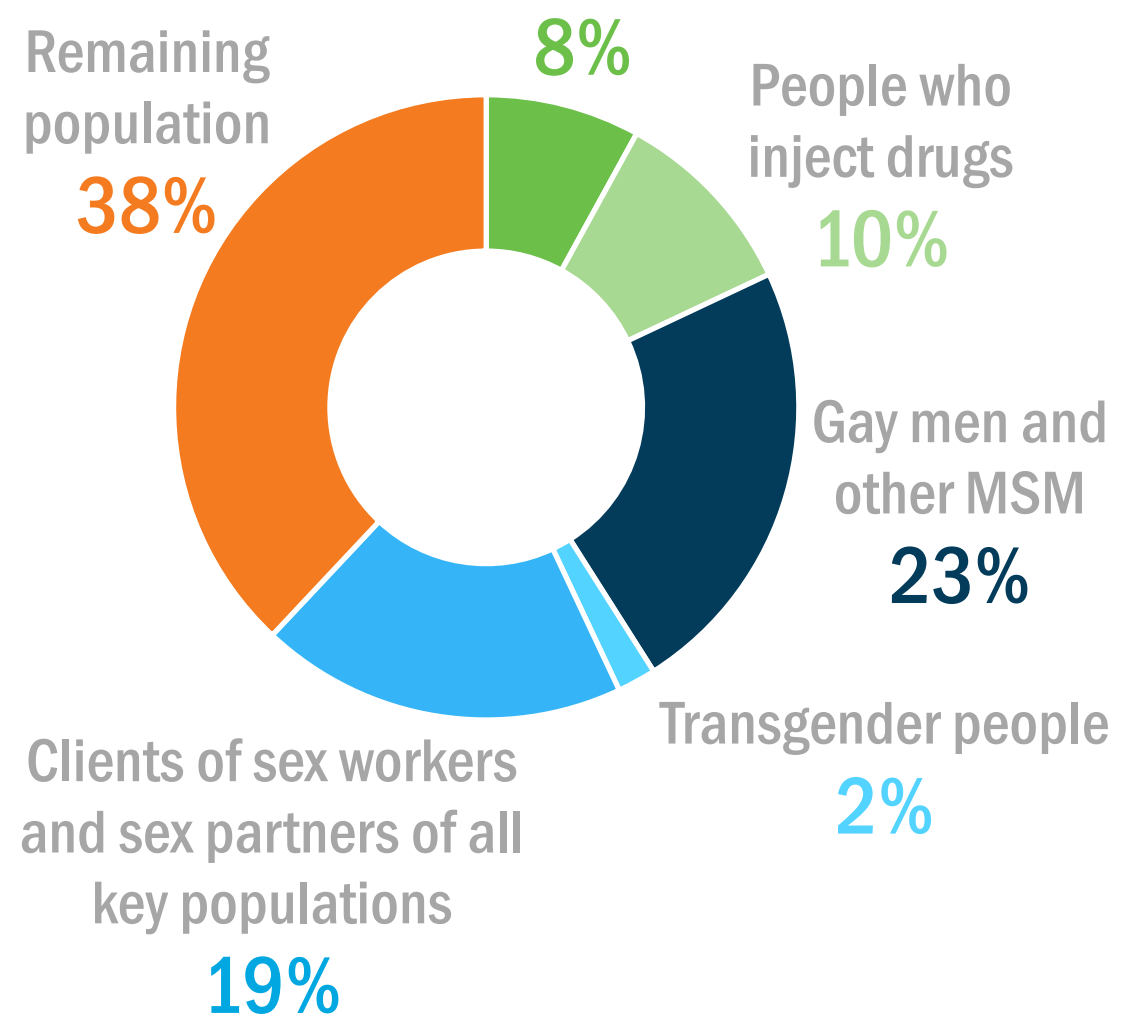





\section{Definitions of Implementation Science (IS)}

- IS as evolving field that includes clinical research but also identifies and addresses barriers to and facilitators of uptake (Bauer \& Kirchner 2020).

- Confronts common challenge of evidence-based intervention not taken/scaled up or when scaled not effective

- Not only to improve program effectiveness / uptake, but also to explain what worked, why, and under what circumstances (Padian et al. 2011).

- Substantial overlap with 'Operations Research' which identifies and diagnoses program problems, tests solutions, and promotes research uptake (Fisher \& Foreit 2002). 


\section{Wide range of study designs/goals}

- Diagnostic assessments to highlight specific risk/need

- Formative research to inform intervention design

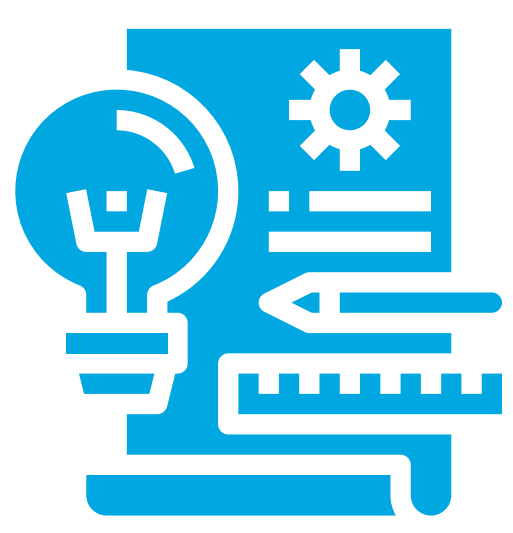

- Process evaluations to assess who is (and isn't) being reached with activities

- Intervention/evaluation studies to test program effects and cost-effectiveness, in one or more contexts 


\section{Research utilization as hallmark of IS}

Key to uptake in programs/policy

- Involvement of stakeholders at all stages of research process

- E.g., study design; data interpretation workshops

- Fosters champions to promote use of findings

- Maximizes relevance and

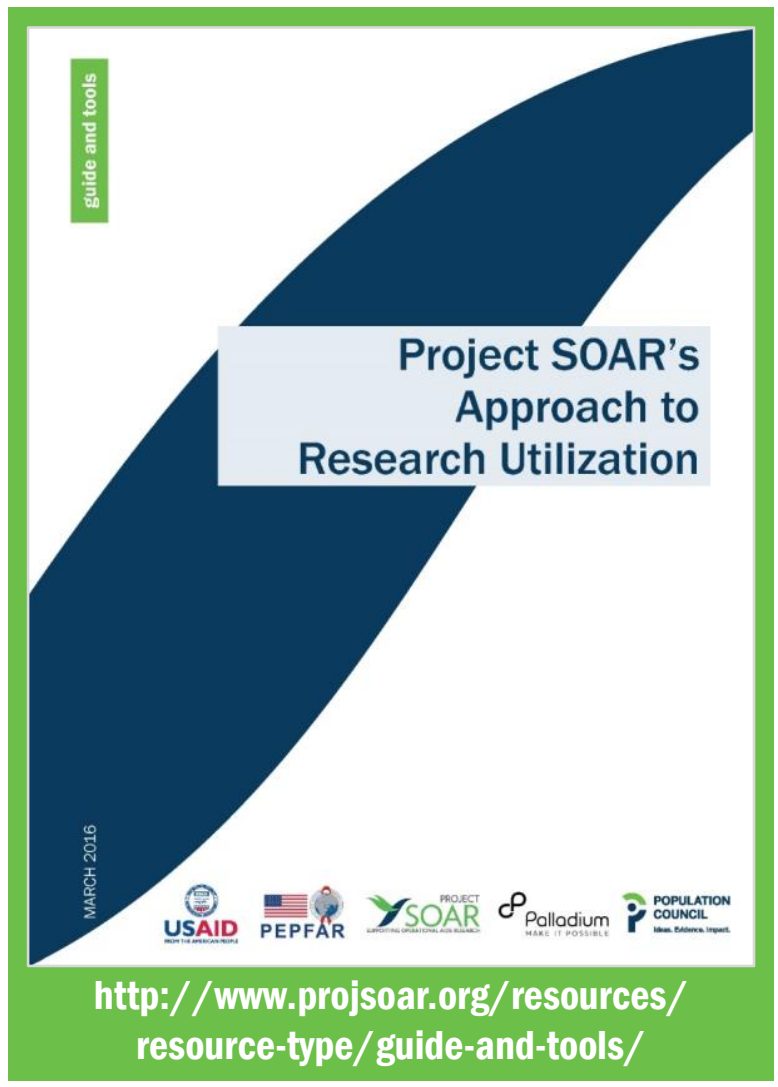
applicability of findings 


\section{Applied research that acknowledges different stakeholder perspectives}
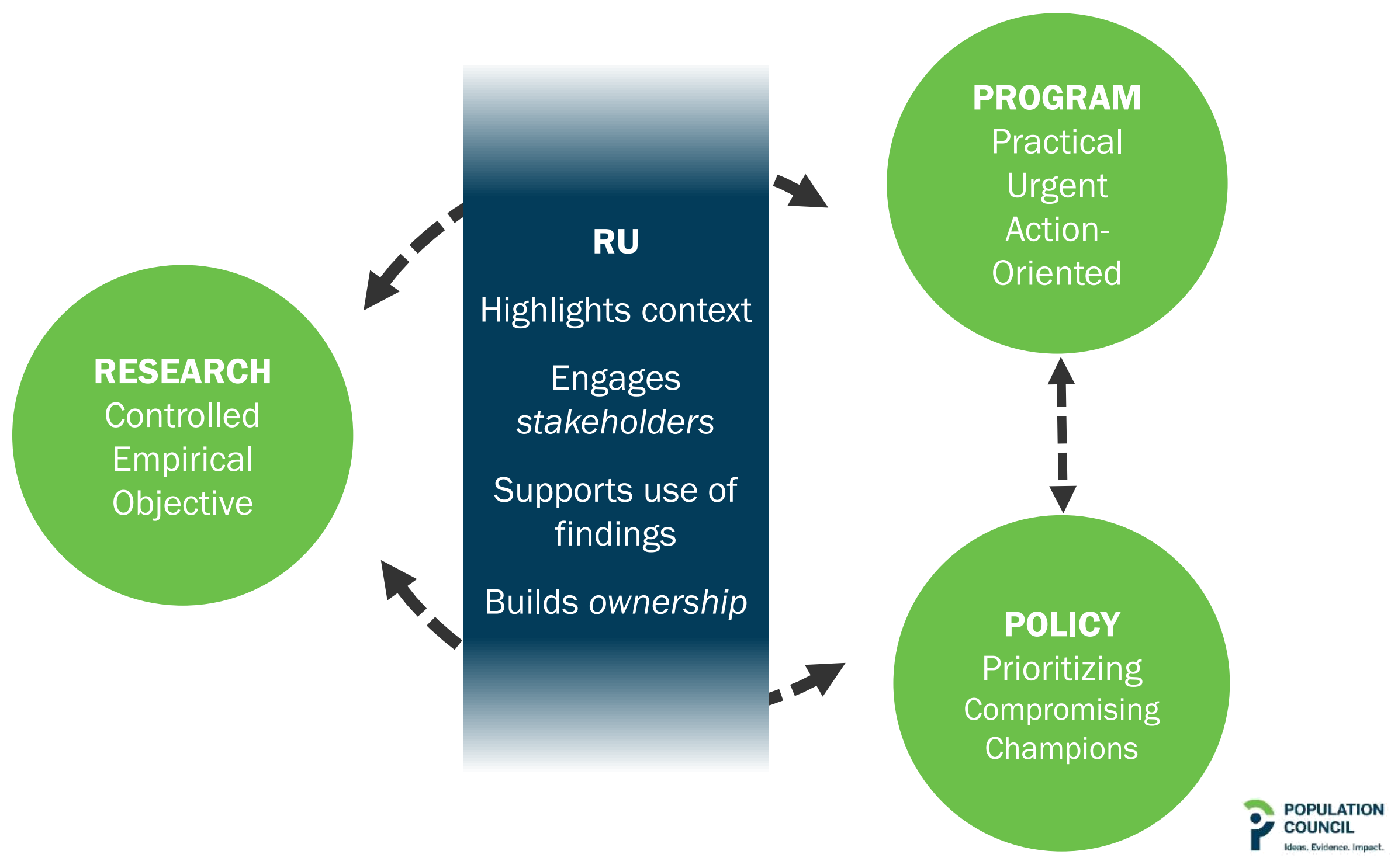


\section{Project SOAR (2014-21)-HIV IS to inform program}

\section{and policy}

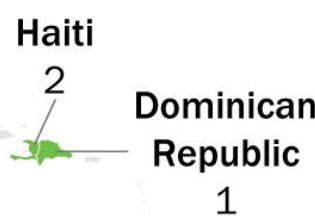

Global Activities

10

2

divire

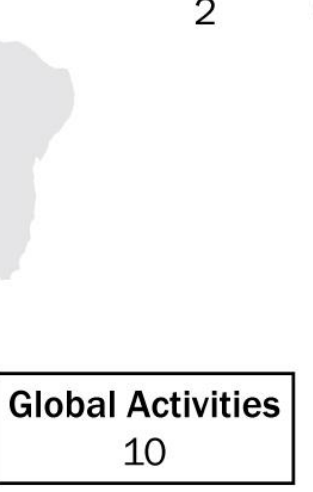

70 studies in 21 countries 


\section{DREAMS IS Research (2016-21)}

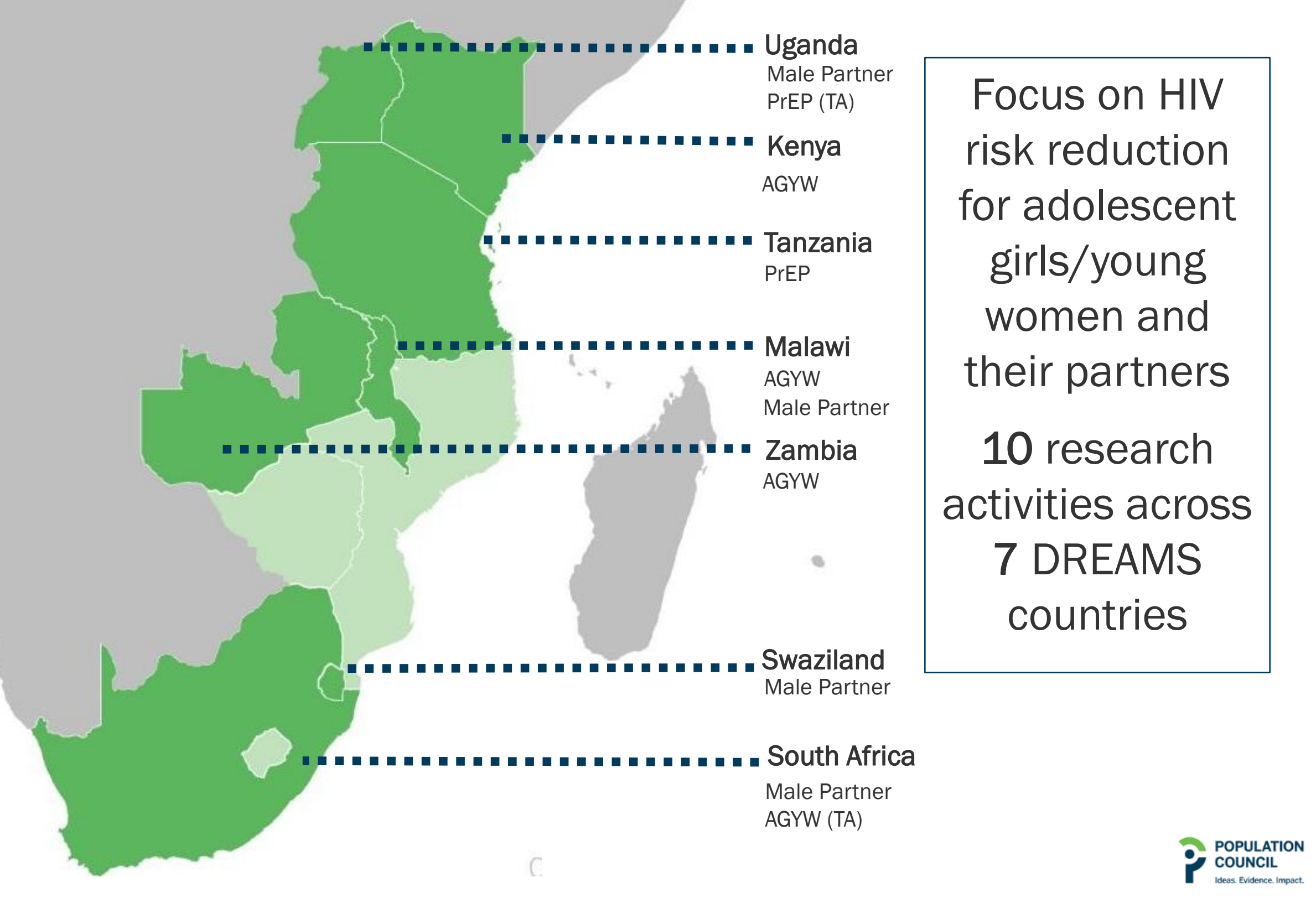




\section{Documenting barriers/facilitators of HIV program success}

- PLHIV Stigma Index: survey tool to quantify stigma and effects

- Used for advocacy, inform service delivery

- Developed by community \& administered to PLHIV by PLHIV

- Examples of stigma: gossip, social exclusion, physical harassment, inadequate/insensitive care 


\section{Effects of multiple and intersecting stigmas}

Stigma experienced by sex workers in the

Dominican Republic $(n=216)$

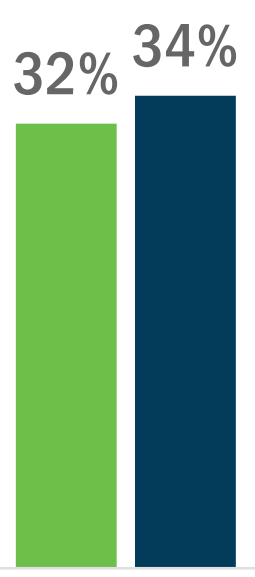

Family members made discriminatory remarks

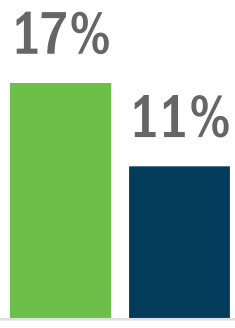

Physically abused

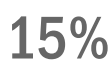

$12 \%$

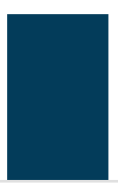

Blackmailed

Verbally harrassed
Sex workers living with HIV in the DR reported stigma in healthcare services significantly more than did other women.

Due to your selling sex

Due to your HIV status 


\section{Internalized stigma is common and impactful}

"I am ashamed that I am HIV positive."

$72 \%$

(example scale item)

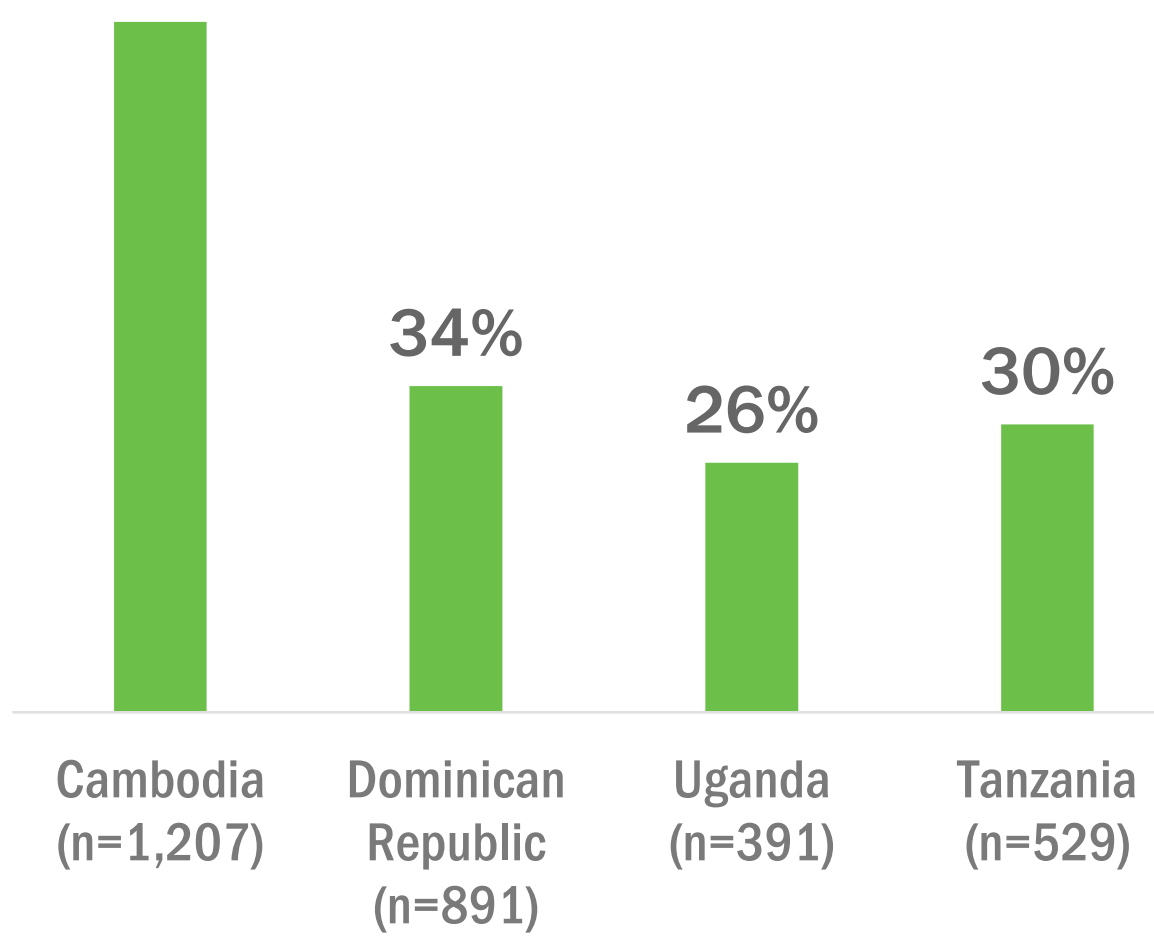

Higher internalized stigma was significantly associated $(p<0.001)$ with:

- Greater depression/anxiety (in all 4 countries)

- Lower current ART use (in the DR and Tanzania) 


\section{Understanding what leads to resiliency}

- Factors at individual, interpersonal, and structural levels affect whether PLHIV report resilience

\begin{tabular}{l|l|c|c|c|}
\hline LEVEL & FACTOR & $\begin{array}{c}\text { CAMBODIA } \\
(n=1,207)\end{array}$ & $\begin{array}{c}\text { DOMINICAN } \\
\text { REPUBLIC } \\
(n=891)\end{array}$ & $\begin{array}{c}\text { UGANDA } \\
(n=391)\end{array}$ \\
\hline INDIVIDUAL & Internalized stigma & $-0.28^{*}$ & $-0.31 * * *$ & $-0.41^{*}$ \\
\hline INTERPERSONAL & HIV-related stigma from close family & 0.10 & $-0.73^{*}$ & -1.44 \\
\hline $\begin{array}{l}\text { STRUCTURAL/ } \\
\text { POLICY }\end{array}$ & $\begin{array}{l}\text { Awareness of legal protections for } \\
\text { PLHIV in community }\end{array}$ & $1.41^{* * *}$ & $0.62 *$ & 0.09 \\
\hline
\end{tabular}

${ }^{*} \mathrm{p}<0.05 * * \mathrm{p}<0.01 * * * \mathrm{p}<0.001$

Beta coefficients from multivariate models, including controls for sociodemographic characteristics 


\section{Identifying who to prioritize reaching, where, and with what}

- Innovative analysis (latent class analysis) for better identification of sub-groups even within high prevalence contexts

- Segmentation based on multiple characteristics

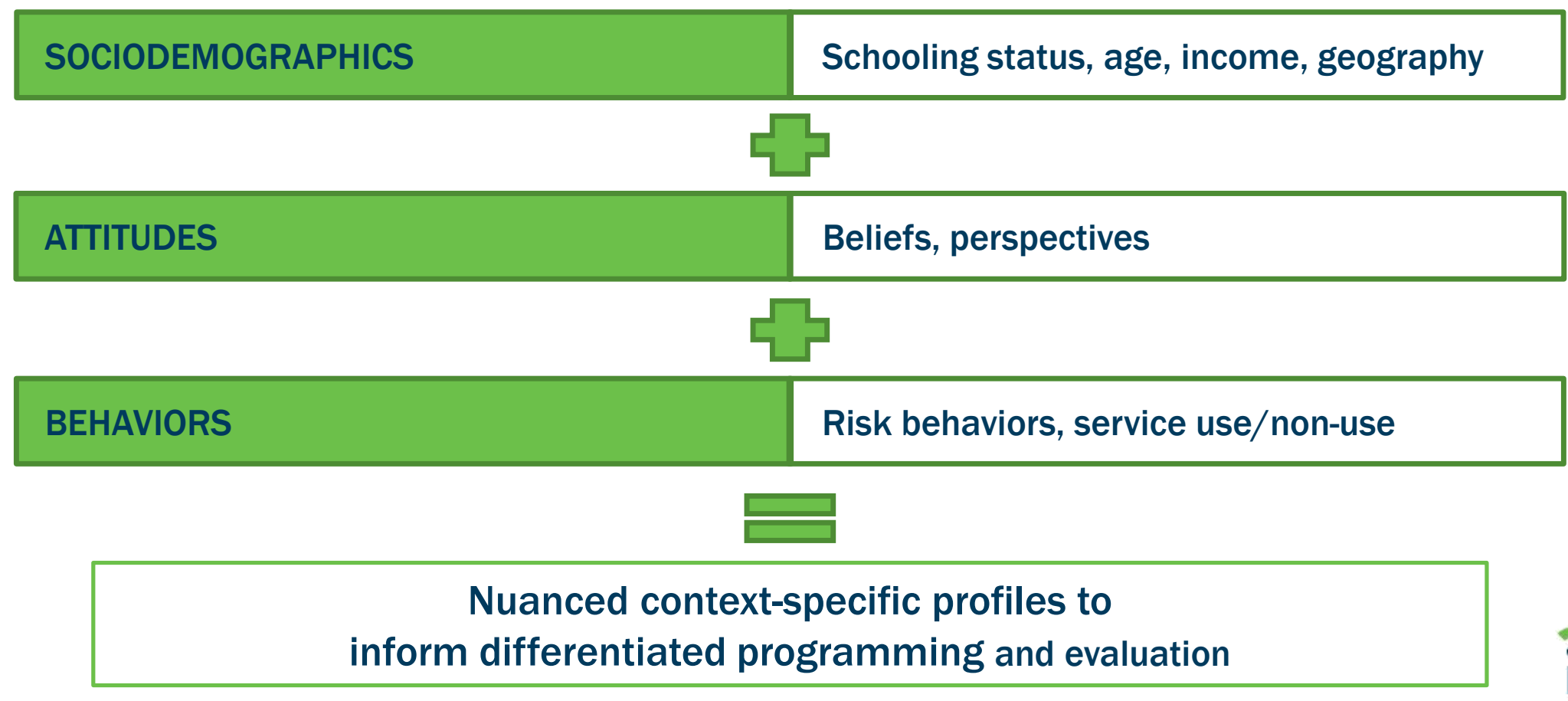




\section{HIV risk profiles to tailor/target programming (men in South Africa; $n=1,846$ )}

- Distinct subgroups/ profiles where not all have equal risk

- Risk profiles of older and younger men don't look the same

- Differentiated programming
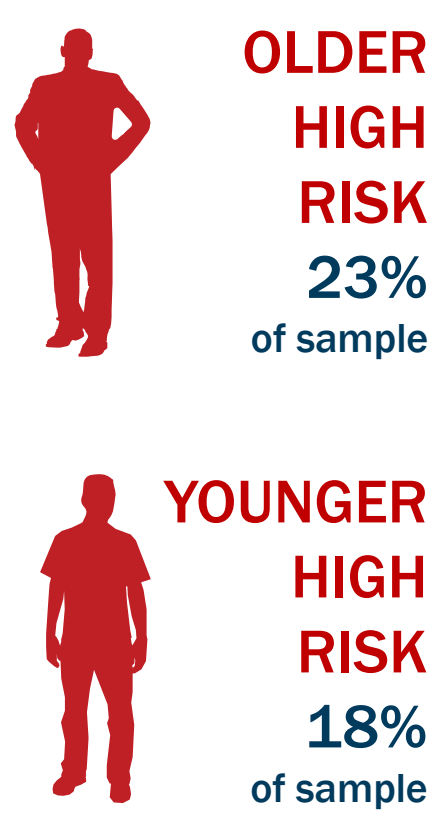

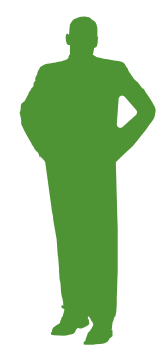

OLDER

LOW RISK $25 \%$

of sample 


\section{Gender attitudes as major distinguishing factor}

Highest-risk group = most gender-inequitable

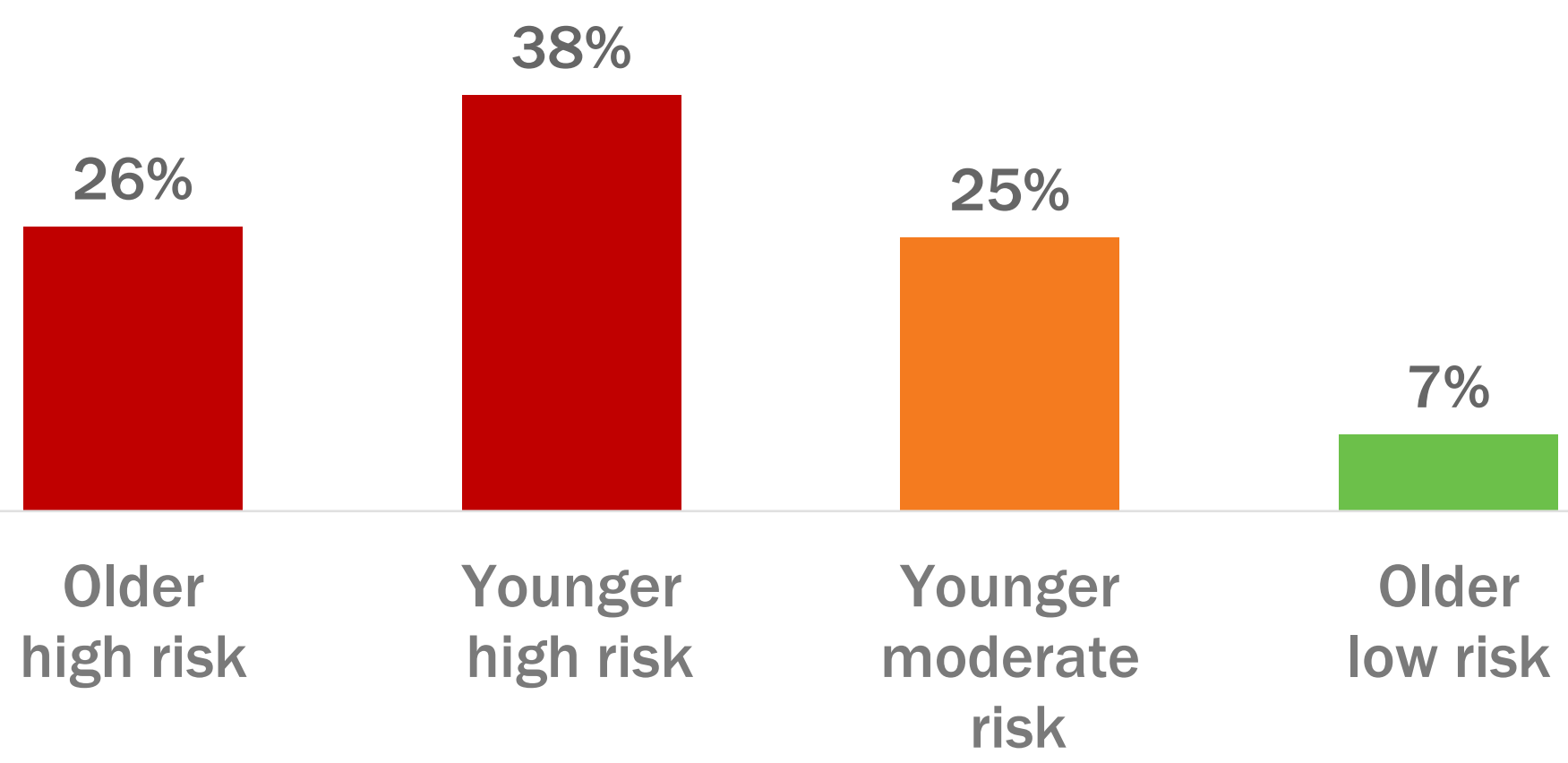

Endorsement of highly inequitable gender norms 


\section{Assessing reach of programming}

- Do programs identify and recruit most at-risk AGYW?

- Cross sectional surveys with AGYW recruited from DREAMS program rosters and within the community

\begin{tabular}{|lccc|}
\hline Country & Kenya & Malawi & Zambia \\
\hline Region & Kisumu County & $\begin{array}{c}\text { Machinga \& } \\
\text { Zomba Districts }\end{array}$ & $\begin{array}{c}\text { Lusaka \& Ndola } \\
\text { regions }\end{array}$ \\
Study sites & $\begin{array}{c}\text { Urban \& peri-urban } \\
\text { communities }\end{array}$ & $\begin{array}{c}\text { Rural } \\
\text { communities }\end{array}$ & $\begin{array}{c}\text { Urban } \\
\text { Sample size }\end{array}$ \\
\hline
\end{tabular}

* Only recruited from DREAMS rosters 


\section{DREAMS package: Combination of "evidence- based" interventions layered in same location}

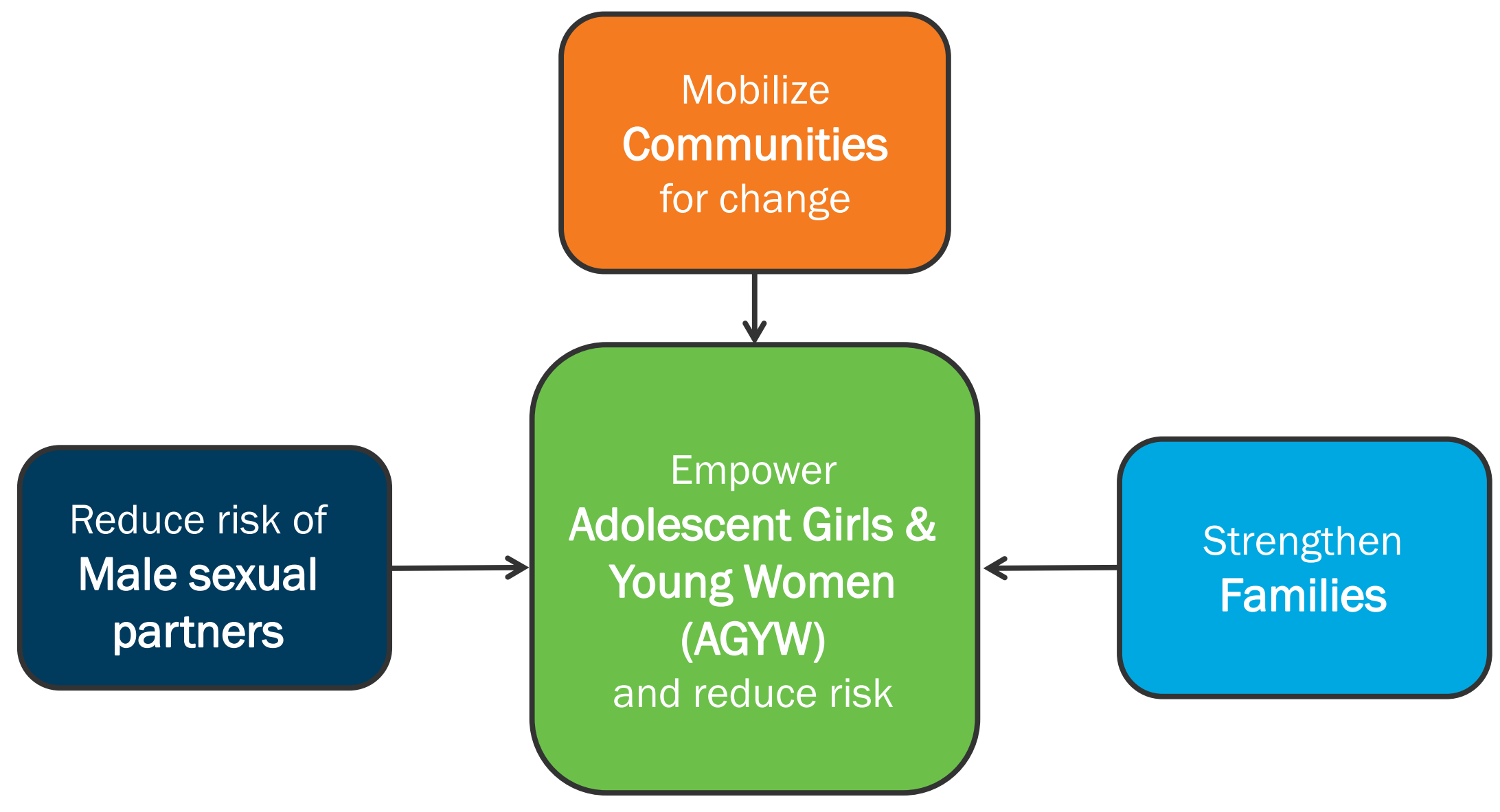




\section{Many at risk reached but gaps remained}

- DREAMS reaching many at-risk AGYW

$\checkmark$ High STI experience

$\checkmark$ Multiple partnerships

$\checkmark$ Experienced sexual violence in the last 12 months

- Some sub-populations of AGYW not as well represented

\begin{tabular}{|lcc|cc|}
\hline & \multicolumn{2}{c|}{ Zambia } & \multicolumn{2}{c|}{ Kenya } \\
& DREAMS & Non-DREAMS & DREAMS & Non-DREAMS \\
\hline Married & 2 & 18 & 19 & $\%$ \\
Sexually active & 41 & 66 & 62 & 78 \\
Alcohol use before sex & 18 & 26 & 10 & 11 \\
Consistent condom use & 24 & 12 & 16 & 9 \\
\hline
\end{tabular}




\section{4a. Effects of innovative intervention: Improving access to treatment among FSWs}

- Reaching key populations with ongoing care/treatment challenging

- Community-based ART promising strategy, but not approved in Tanzania

- Study to test feasibility/ acceptability, and effectiveness of bringing HIV treatment to sex

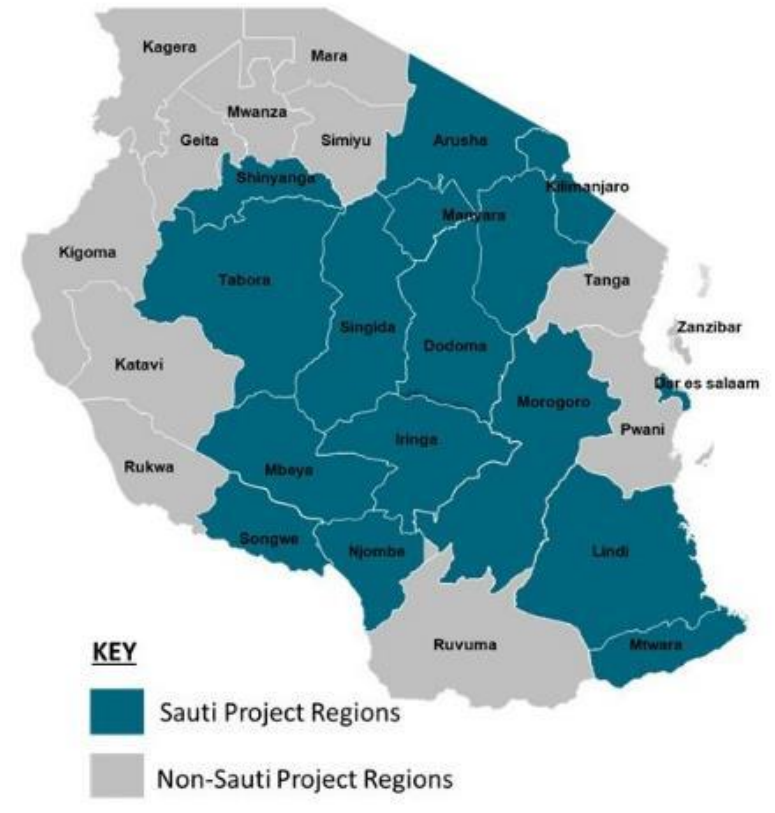
workers in community settings (e.g., mobile clinics, brothel-based) 


\section{Methods and sample}

Mixed-methods, quasi-experimental prospective cohort design

\begin{tabular}{|c|c|c|}
\hline & $\begin{array}{l}\text { Intervention } \\
\text { (Njombe) }\end{array}$ & $\begin{array}{l}\text { Comparison } \\
\text { (Mbeya) }\end{array}$ \\
\hline Baseline & $\mathrm{n}=309$ & $\mathrm{n}=\mathbf{3 0 8}$ \\
\hline Interviewed at 6 months & $256(83 \%)$ & $253(82 \%)$ \\
\hline Interviewed at 12 months & $265(86 \%)$ & $262(85 \%)$ \\
\hline $\begin{array}{l}\text { Participants interviewed at } \\
\text { BOTH } 6 \text { \& } 12 \text { months }\end{array}$ & $246(80 \%)$ & 234 (76\%) \\
\hline $\begin{array}{l}\text { Participants who had viral load (VL) } \\
\text { taken at: }\end{array}$ & $\begin{array}{l}84 \% \text { ( } 6 \text { months }) \\
76 \% \text { (12 months) }\end{array}$ & $\begin{array}{l}96 \% \text { (6 months) } \\
87 \% \text { (12 months) }\end{array}$ \\
\hline
\end{tabular}




\section{Community-based services successful in reducing HIV treatment barriers}

ART initiation

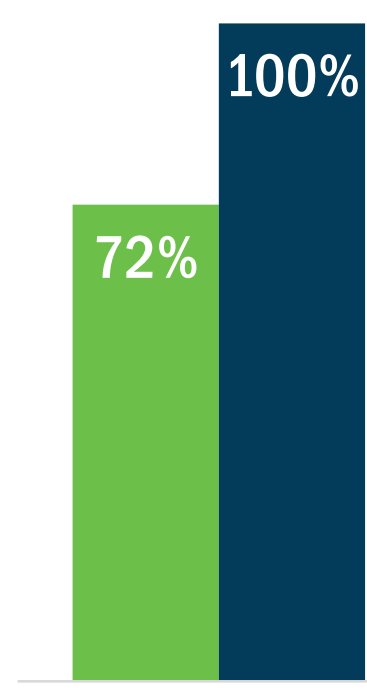

Midline

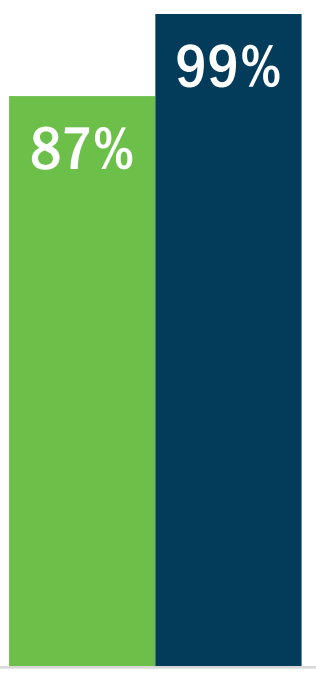

Endline

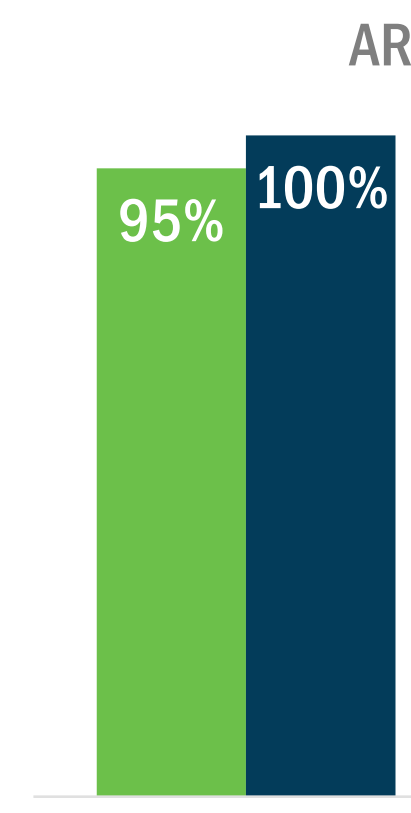

Midline
ART retention

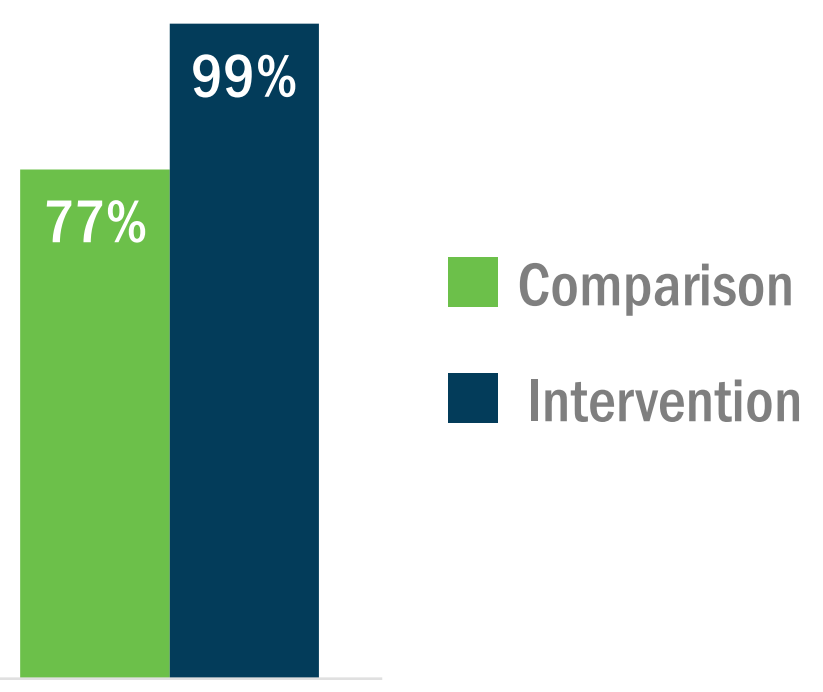

Endline

Government of Tanzania used findings from this study with sex workers to inform national ART guidelines. 


\section{4b. Effects of innovative interventions: gender focused community-based programming to improve HIV service use}

- At baseline, endorsement of inequitable gender norms associated with lower odds of treatment (ART) use

\begin{tabular}{|lcc|}
\hline & HIV-positive & HIV-positive \\
& WOMEN & MEN \\
& $(n=122)$ & $(n=48)$ \\
GEM Scale (mean score, 23 items) & aOR & a. \\
Higher=more inequitable & $0.2 * *$ & 0.6 \\
Men's violence and control over women & $(0.1,0.5)$ & $(0.1,3.8)$ \\
Men as decision-maker in a couple & $0.3 *$ & 1.1 \\
& $(0.1,1.0)$ & $(0.2,5.4)$ \\
Men's toughness and avoidance of help-seeking & $0.2 * *$ & $0.3 *$ \\
& $(0.1,0.5)$ & $(0.1,0.9)$ \\
& $0.4 *$ & 0.8 \\
& $(0.1,1.0)$ & $(0.2,4.1)$ \\
\hline
\end{tabular}

$* p<0.05 * * p<0.01 * * * p<0.001$; Controlling for age, marital status, education 


\section{Population-level shifts in gender norms found}

- Cluster randomized controlled trial of Tsima showed large increases in support for equitable gender norms in both intervention and control communities

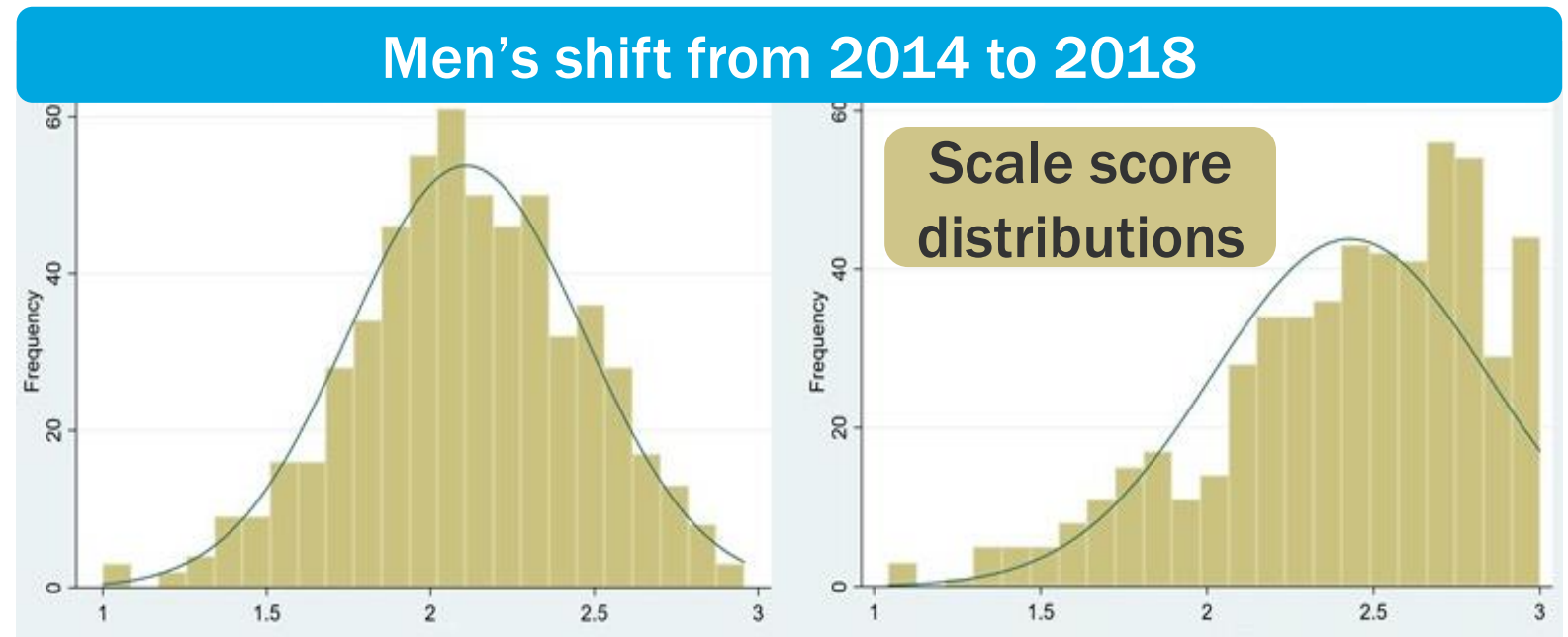

Qualitative research suggested shifts were influenced by recent, rapid increase in access to media (satellite TV, smartphones) 


\section{Intervention led to decreases in partner violence}

- Among women ages 18-29, the intervention was associated with half the odds of IPV

- Adj. Odds Ratio 0.48 ( $p<0.05)$

- Qualitative findings:

- Reduced IPV in intervention villages was attributed to couples learning to communicate more constructively through Tsima

- Broader shifts in norms may have been critical enabler of reduced IPV

I was not communicating with her...She was always complaining about it, arguing and sometimes I was abusing her physically when she complained, but Tsima has changed that, we always communicate nowadays.

-Male community member

Note that analyses still underway for effects on HIV testing and treatment outcomes. 


\section{Concluding thoughts...}

- Still notable challenges to address before global and US-based HIV epidemic under control

- Many similar issues put people at risk of HIV and inhibit service use/program success both globally and in the US-

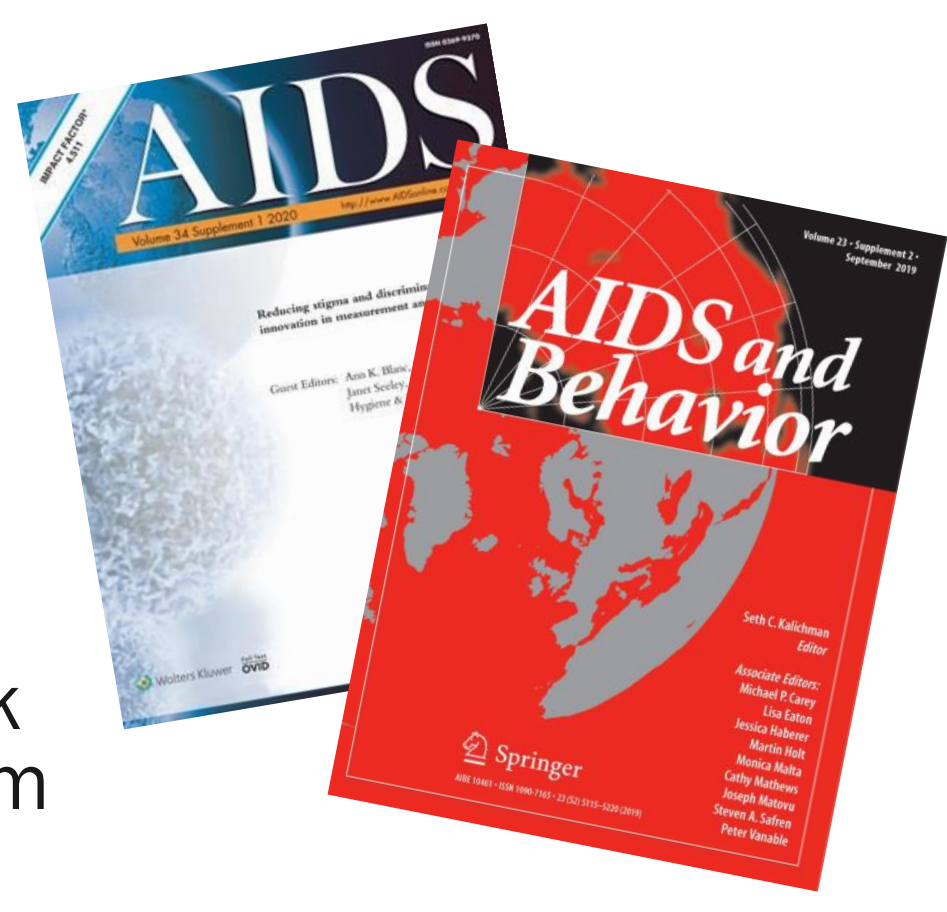
e.g., stigma, healthcare access, gender dynamics

- Lessons learned and strategies used globally can be applied in the US

- E.g., Gender-transformational program for young men in Pittsburgh

- Miller, E., K. A. Jones, A. J. Culyba, T. Paglisotti, N. Dwarakanath, M. Massof, Z. Feinstein, K. A. Ports, D. Espelage, J. Pulerwitz, A. Garg, J. Kato-Wallace, K. Z. Abebe. (2020) Effect of a community-based gender norms program on sexual violence perpetration by adolescent boys and young men: A cluster randomized clinical trial. JAMA Netw Open. 3(12):e2028499. 


\section{ACKNOWLEDGMENTS}

- Enda Santé: Daouda Diouf, Fatou Mbacké Sy

- EpiCenter: Cherie Cawood

- Global Fund: Noah Metheny (formerly USAID)

- GNP+: Julian Hows, Omar Syarif

- ICW: Florence Anam, Sophie Brion, Margaret Happy

- Jhpiego-Sauti: Caterina Casalini, Gasper Mbita, Albert Komba

- JHU: Stefan Baral, lliassou Mfochive Njindam, Oluwasolape Olawore, Gnilane Turpin, Fan Yang

- Kenya DREAMS Implementing Partners: PATH/APHIAplus

- Metabiota: Guy Fako, Ubald Tamoufe

- NACP Tanzania

- NAFOPHANU: Richard Batamwita, Stella Kentutsi, Richard Mugumya, Prossy Nanyanzi, Enos Sande, Stephen Sentongo

- National Institute of Medical Research, Tanzania: Kidola Jeremiah

- Population Council: Julie Pulerwitz; Scott Geibel, SOAR Proj Dir; Sanyukta Mathur, DREAMS Proj Dir; Lou Apicella; Barbara Friedland; Ann Gottert; Nicole Haberland; Craig Heck; Sherry Hutchinson; Nrupa Jani; Samuel Kalibala (Palladium); Deanna Kerrigan (GWU); Tracy McClair; Mike Mbizvo; Maurice Musheke; Jerry Okal; Sangram K. Patel; Nanlesta Pilgrim; Vaibhav Saria; Waimar Tun, Lung Vu, Deborah Weiss; Ellen Weiss; Eileen Yam

- PEPFAR Kenya

- PEPFAR OGAC

- PEPFAR Zambia
- RTI: Laura Nyblade, James Wrenn

- UNAIDS: Chris Mallouris, Laurel Sprague

- USAID/Global: Ugo Amanyeiwe, Alison Cheng, Aasha Jackson, Amelia Peltz, Sarah Sandison, Cameron Wolf, Aisha Yansaneh

- USAID/South Africa: Virginia Francis

- USAID/Tanzania: Todd Koppenhaver, Erick Mlanga

- University of California San Francisco: Sheri Lippman

- University of North Carolina: Audrey Pettifor

- University of the Witwatersrand: Kathleen Kahn

- Zambia DREAMS Implementing Partners: PACT, CMMB, MSZ

- Study participants

- With funding support from

- Bill \& Melinda Gates Foundation

- PEPFAR and USAID

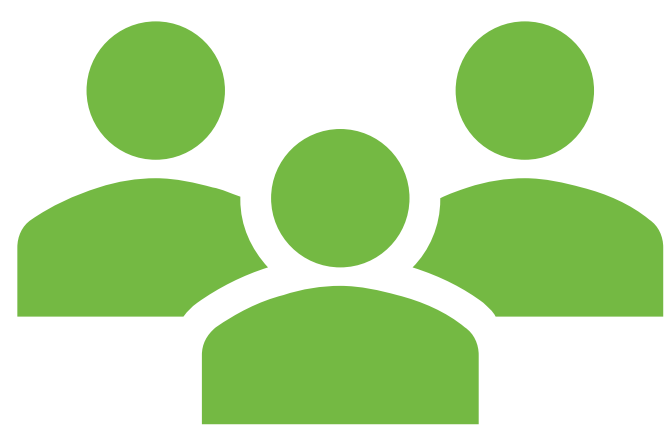




\section{Partners and donors}
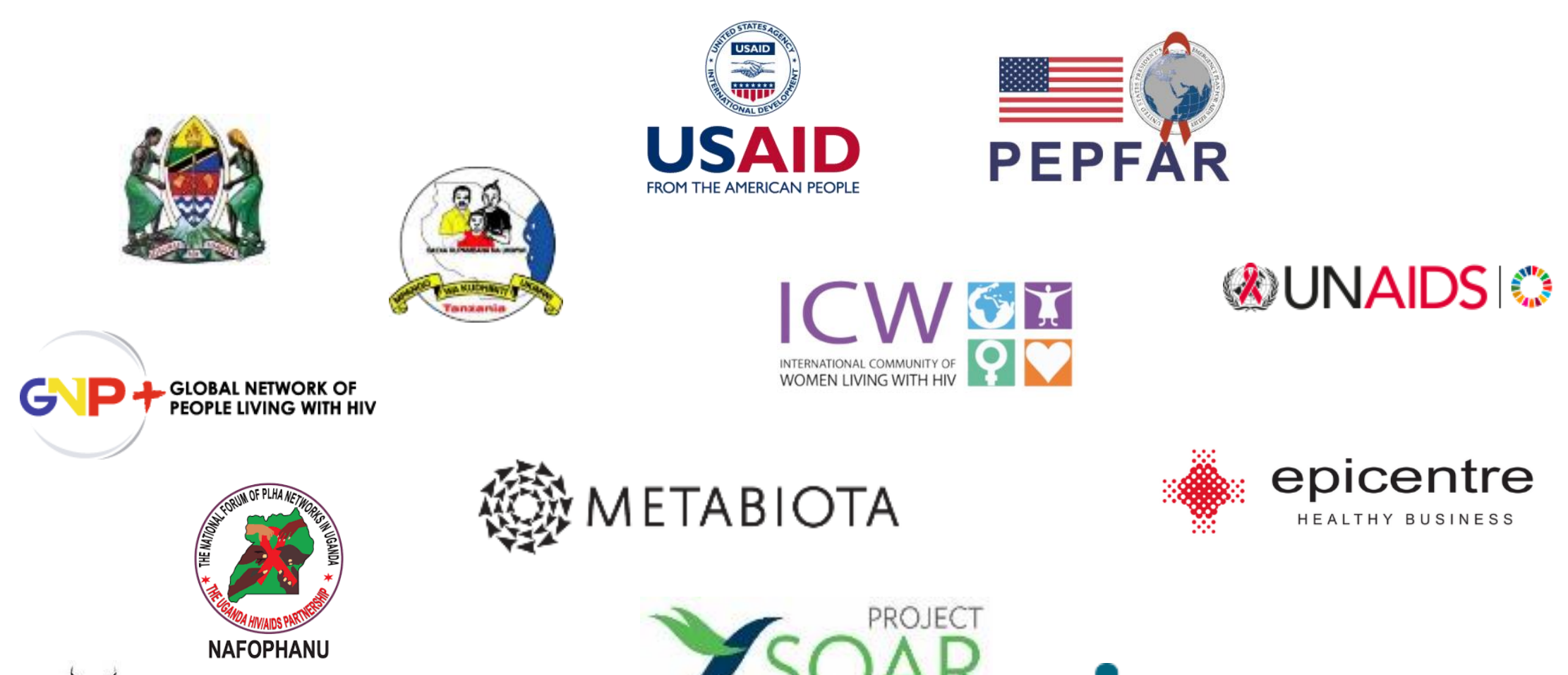
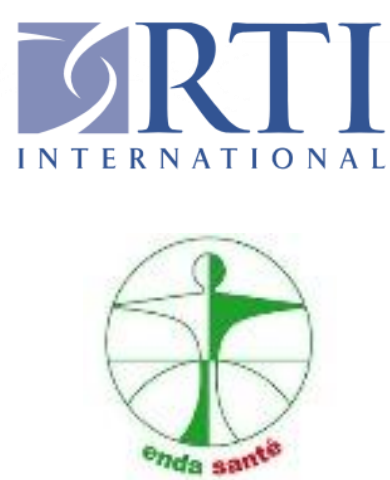

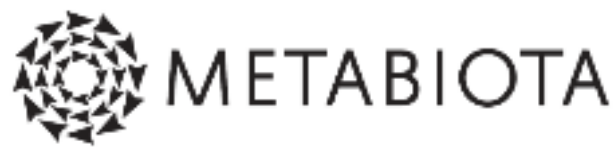

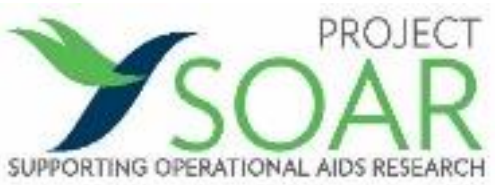

盘

JOHNS HOPKINS

BLOOMBERG SCHOOL

of PUBLIC HEALTH

\section{epicentre}

HEALTHY BUSINESS
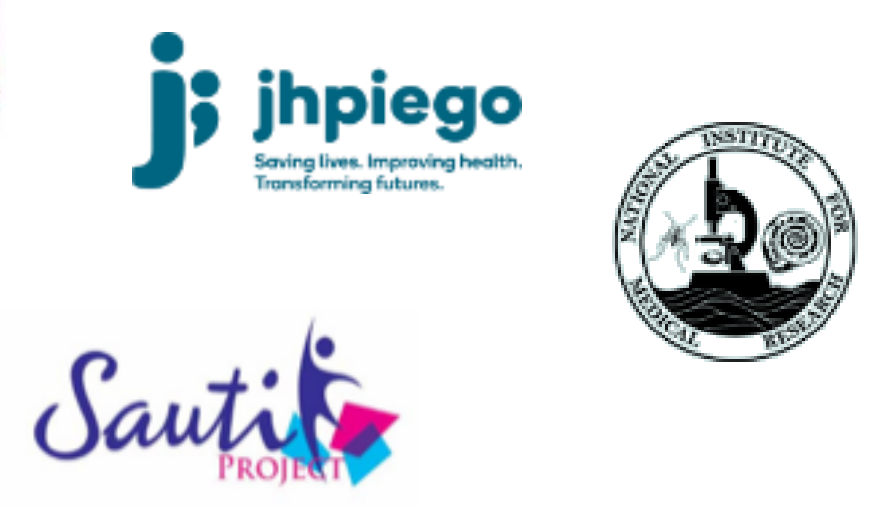

POPULATION 


\section{POPULATION}

\section{COUNCIL}

Ideas. Evidence. Impact.

\section{THANK YOU!}

信pubinfo@popcouncil.org

popcouncil.org

@Pop_Council 